\title{
Teknokultura
}

ISSNe: 1549-2230

http://dx.doi.org/10.5209/TEKN.57269

\section{Tecnologías emergentes, redes al acecho de la sociabilidad}

\author{
Luis Gregorio Iglesias Sahagún"; Rosario González Arias²
}

Recibido: 19 de septiembre de 2017 / Revisado: 7 de diciembre de 2017 / Aceptado: 26 de diciembre de 2017. Open peer reviews

Resumen. Este trabajo destaca algunos síntomas notorios en múltiples sociedades a partir de la instalación de las "tecnologías emergentes", en particular las tecnologías de la información y la comunicación. Apoyados en la idea de que esa capacidad tecnológica está provocando una recomposición de la cultura en un neocapitalismo conexionista, señalamos que además de la constricción del campo de lo posible operada por el sistema de una economía de mercado central y monetizado, los artefactos y dispositivos tecnológicos-particularmente los portátiles - están ejerciendo una nueva constricción, por la vía de una re-organización de ese campo de lo posible. Encontramos signos inquietantes de pérdidas de la autonomía, una reducción de los márgenes para el ejercicio de ésta por parte de los individuos y las colectividades. La situación termina de complicarse cuando vemos que en las sociedades con una capacidad instalada de tecnologías de la comunicación y el transporte, efectivamente se prodigan las formas reticulares en múltiples ámbitos: producción, provisión de servicios (shared economy), vecindario, escuela, universidad, etc. "La red", como metáfora, transmite mucho de lo que sucede, y cómo, en nuestras vidas cotidianas. Concebimos la sociabilidad como la forma que pueden adoptar las concurrencias, la forma del "estar haciendo algo juntos", las formas del "poner en común algo", las formas del "hacer en torno y a propósito de ese algo puesto en común", planteamos la hipótesis de que con las tecnologías emergentes y la reticulación social, se ha puesto también en marcha un vector de componente centrífugo que bloquea, dificulta o inhibe la sociabilidad.

Palabras clave: autonomía; estandarización; macrodatos; minería de datos; multitarea.

\section{[en] Emergent Technologies, networks that lurks the sociability}

Abstract. This paper highlights several symptoms that can be observed in multiple societies since the installation of so called "emergent technologies", especially technologies of information and communication. Supported on the idea that this technological capacity provokes an alteration of the culture, which becomes a connectionist neo-capitalism, we suggest that besides the constriction of the "field of the possible" produced by the system of a central market economy, the appliances and technological devices - the portable ones particularly - exerts a new constriction and, actually, reorganization, of this "field of the possible" ". We find this as disturbing signs of loss of autonomy, such as a reduction in margins for the exercise of autonomy by individuals and collectivities. We will argue that the situation becomes sophisticated in societies with a high installed capacity of communication, information and transportation technologies. In such societies, in fact, reticular forms are lavished in multiple areas: production, provision of services (shared economy), neighborhood, school, university, etc. No doubt "the network" is the metaphor that best conveys much of what happens and the ways it happens in our daily lives. Starting from a conception of sociability, understood as the form that concurrences can adopt, the form of "doing something together", the forms of "putting something in common", the forms of "doing about that something put in common ", we hypothesized that with

\footnotetext{
$1 \quad$ Universidad Autónoma de Querétaro (México)

E-mail: gregoriux@posteo.net

2 Universidad Autónoma de Querétaro (México)

E-mail: charogonza@yahoo.es
} 
emergent technologies and social reticulation, a vector of centrifugal component that blocks, hinders or inhibits sociability has also been set in motion.

Keywords: autonomy; big data; data mining; multitask; standardization.

Sumario: 1. Introducción. 2. Excurso sobre la sociabilidad. 3. Tecnologías emergentes y escalas de la desmesura. 4. Estrecho cerco a la autonomía. 5. Concluyendo por ahora. 6. Referencias.

Cómo citar: Iglesias Sahagún, L.G.; González Arias, R. "Tecnologías emergentes, redes al acecho de la sociabilidad": Teknokultura, vol. 15 (1) 2018, pp. 119-137.

Matthew Arnold, que foi (embora aquí se não saiba, o que quer dizer apenas que aquí se não sabe) um dos grandes poetas do século dezanove, definiu, numa frase que ficou célebre, a nulidade íntima de civilização puramente material, "De que te serve um comboio que te leva num quarto de hora de Camberwell para Islington, se te leva de uma vida reles e estúpida em Camberwell para uma vida reles e estúpida em Islington?” [Fernando Pessoa (c. 1923) en Barreto 2015]

\section{Introducción}

Qué difícil es ir a contrapelo del imaginario de una época. Entiéndase, del imaginario instituido, y aún hegemónico. Castoriadis (2007) propone distinguir básicamente entre un imaginario instituido, que es aquel que tiene vigencia en significaciones sancionadas positivamente por una sociedad, y cristaliza por tanto en instituciones, en prácticas, en arquitecturas, en organizaciones; comprometido con hacer durar la institución de esa sociedad (institución de instituciones) tal como se halla en un momento dado. Y por otro lado un imaginario radical, que es uno que suele presentarse, en su proceso mismo de "fraguarse", en sus ensayos y tanteos, a veces osados, a veces fugaces; es el momento instituyente, del significar o resignificar abriendo nuevas rutas de sentido en una sociedad en una época determinada.

Aludimos a la dificultad de ir en contrasentido del imaginario de una época porque en este artículo nos proponemos exponer algunas reflexiones críticas de una situación en la que, tememos, estamos en muchas sociedades, y es la de encontrarnos en la condición de "rehenes del desarrollo del mercado de la tecnología".

Reflexionamos sobre el auge presente de los Big Data, las Data Mining, el P2P y la creciente penetración de las redes sociales en todos los rincones de las sociedades. Lo que nos lleva a plantear que, además de ese "secuestro" de la vida social en la economía de mercado, en la época presente, con sus condiciones de desarrollo técnico y su saber tecnológico, está ocurriendo un segundo secuestro que acarrea el efecto concomitante de un mayor estrechamiento del cerco a las posibilidades de la autonomía, tanto en su amplitud colectiva como individual.

Además, nos arriesgamos a decir, todo ello puede restringir y dificultar seriamente la ocurrencia y despliegue de la sociabilidad, asunto por el cual nos vimos directamente concernidos a participar con esta colaboración en el número de Teknokultura. $\mathrm{Y}$ es que nosotros comenzamos una investigación sobre las prácticas de sociabilidad entre jóvenes universitarios (en Querétaro, México), y al poco tiempo de haber comenzado el trabajo empírico, mediante entrevistas y grupos de discusión, nos vimos 
llevados a un punto en el cual aquello de lo que hablábamos, leíamos y comenzamos a escribir eran mensajes de texto, informaciones y cotilleos que circulan en las redes sociales, así como maneras de "conducirse" en ellas. Así, decidimos corresponder a la invitación de los editores y compartir algunas reflexiones que se producen siempre a través del prisma de la sociabilidad.

\section{Excurso sobre la sociabilidad}

En efecto, ya en la inquietud inicial de nuestro proyecto figuraba el tópico de las "nuevas tecnologías", cómo eran utilizadas y las consecuencias que podrían traer para las prácticas de sociabilidad entre los jóvenes universitarios. El objetivo de nuestro proyecto, podemos decir que el principal, es el de explorar la posible relación entre las prácticas de sociabilidad de los jóvenes con los modos en que significan dos términos: política y ciudadanía. Hasta el momento hemos realizado el trabajo empírico consistente en la producción intencionada de textos mediante las técnicas de entrevista abierta semiestructurada y grupo de discusión. También seguimos en el trabajo de discusión y reconstrucción conceptual de la categoría de sociabilidad. Una vez dicho esto, no sobra explicitar que siguiendo nuestro diseño empírico hemos trabajado, entre diciembre de 2016 y enero de 2017 tres grupos de discusión, constituidos por estudiantes universitarios en un rango etario de 20 a 26 años, de diez facultades de la Universidad Autónoma de Querétaro. También, hasta el momento se han hecho siete entrevistas abiertas semiestructuradas con los mismos perfiles que para los grupos de discusión; debemos advertir que el material textual no está aún analizado. Sin embargo, a partir de lo escuchado en los grupos de discusión, y vuelto a escuchar en el proceso de transcripción para establecer el texto escrito, nos dimos clara cuenta de lo que previamente era sólo una suposición e intuición. Esto es, el papel tan destacado y aún estratégico que llegan a cumplir las nuevas tecnologías en el desempeño o desenvolvimiento $-\mathrm{O}$ no- de la sociabilidad y sus situaciones. ¿Qué queremos decir con esto?

Acordamos con González (2009, p. 296) en que: “Con la palabra sociabilidad la primera dificultad proviene de la presunta transparencia de la noción que facilita el sentido común: sociabilidad sería el atributo de los seres humanos en sociedad". Con lo cual, y dado que queremos poner las cosas más allá del sentido común, van estas precisiones conceptuales del término sociabilidad. Hay registros de la palabra ya en el siglo XVII (Iglesias, 2017) y figura recogida por Jaucourt (1765). A principios del siglo XX, Simmel (1971) introdujo la noción de sociabilidad, concretamente fue en Frankfurt en 1910, en su discurso de apertura del Coloquio de la Sociedad Alemana de Sociología bajo el título de «sociología de la sociabilidad». Posteriormente le consagraría un capítulo de libro "La sociabilidad, ejemplo de sociología pura o formal" (Simmel, 1971). Lo que el autor alemán presenta como sociabilidad es algo concernido con la fenoménica de la sociación; habría que precisar, "la fenoménica de una parte de" la sociación. Se trata del sentimiento gozoso de la asociación misma, del propio estar enlazados, con independencia de los contenidos (intereses y necesidades) puestos en juego para y a través de esa misma interacción; y es que el propio Simmel (1981) ha definido la "sociación" como "la forma que se realiza siguiendo innumerables maneras diferentes, gracias a las cuales los individuos, en virtud de sus intereses, conforman una unidad en el seno de la cual esos intereses se realizan" 
(Simmel, 1981, p. 122). Esto es, que mediante la sociación, por ella y a través de ella, los seres humanos hacemos y mantenemos vigente la sociedad. Ahora bien, en cuanto a la sociabilidad, se trataría de hacer abstracción de los contenidos, para asistir al registro del sentimiento y la satisfacción de la "sociación" pura, del interaccionar con el otro sin una finalidad exterior a esa interacción misma. La sociabilidad sería el evento en que los intereses se dejan, al menos aparentemente, a un lado, lo cual lleva al autor a definirla como la "forma lúdica de la sociación" (Simmel, 2002, p. 197). El único fin de la sociabilidad es la asociación misma. Se trata, pues, de cuando los modos concretos del trato social, cuando la gimnasia de los gestos y las palabras, no están motivadas desde un fin heterónomo de la relación misma. Es decir, cuando ese trazo y esa gimnasia se dibujan y fluyen autonomizados de todo código que no sea el que ellos mismos definen. Es la sociabilidad en tanto que forma autónoma de la sociación.

Avanzado el siglo XX, Gurvitch (1963) vino a formular su consideración sobre la sociabilidad de una manera, si se puede, más abstracta que Simmel. Este académico ruso, avecindado en Francia, ubica la sociabilidad en el nivel intermedio del diapasón de observación sociológica entre lo "macro" y lo "micro"; "agrupamiento específico" le llama a ese nivel, y lo piensa como un microcosmos de las manifestaciones de la sociabilidad. Para Gurvitch (1963) se trata con ella de un fenómeno social total, exterior a los individuos, que podría definirse como las condiciones de posibilidad que habilitan la vinculación dentro de un todo con cualquiera de sus partes. Ahora bien, no obstante esa exterioridad, la sociabilidad perfila y confiere a los individuos atributos que componen y re-componen precisamente su forma de individuos. Y ya a finales del mismo siglo XX otros dos sociólogos nos regalan una práctica y condensada caracterización de nuestra huidiza noción: "la sociabilidad corresponde a lo que hay de formal en una "asociación», la forma del intercambio (tacto, cortesía, etc.) que pesa sobre su contenido" (Degenne y Forsé, 1994, p. 35).

Nosotros entendemos la sociabilidad como una forma o situación. ¿De qué? de las concurrencias. La sociabilidad alude a la forma que pueden adoptar las concurrencias, la forma del "estar haciendo algo juntos", las formas del "poner en común algo", las formas del "hacer en torno y a propósito de ese algo puesto en común", las formas del trato y correspondencia entre personas.

Acción recíproca o lazo de reciprocidad liberado de toda constricción - que contiene en sí misma su propio fin — la sociabilidad en Simmel (1971) sería la expresión prístina del vínculo social. Prístina en el sentido de que es constituyente de ese vínculo social y además en modo redoblado, ya que es confirmatoria de las posiciones de individuos, aquellos que interactúan y se afectan recíprocamente. La tonalidad gozosa y el sentimiento de satisfacción que cualifica la sociabilidad (con independencia y más allá de los contenidos puestos en juego en la sociación) se finca en el mero hecho de que el individuo se asocia con otros y que su aislamiento se resuelve en el acompañamiento, en una unión con otros (Simmel, 1971).

\section{Tecnologías emergentes y escalas de la desmesura}

La expresión Big Data, tomada del inglés y trasladada y puesta tal cual en las lenguas españolas y latinoamericanas, junto con otras denominaciones procedentes del campo de las tecnologías emergentes, es un analizador de situaciones sociales, por- 
que nos da pistas del papel protagónico e impregnante que desempeñan el mundo de los negocios, el comercio internacional, las telecomunicaciones, la automatización, en la cultura vigente y al alza, a la que podríamos referir con rótulos como los de "sociedad de la información", "sociedad red", "sociedad del conocimiento", o "sociedad digital".

Pues bien, por Big Data se alude al almacenamiento y manipulación de grandes volúmenes de datos (estructurados, no estructurados y semiestructurados), que

Tomaría demasiado tiempo y sería muy costoso cargarlos a una base de datos relacional para su análisis. De tal manera que, el concepto de Big Data aplica para toda aquella información que no puede ser procesada o analizada utilizando procesos o herramientas tradicionales. Sin embargo, Big Data no se refiere a alguna cantidad en específico, ya que es usualmente utilizado cuando se habla en términos de petabytes y exabytes de datos. (Barranco, 2012, s.p.)

En cuanto al Data Mining, también relativo al campo de la estadística, es una modalidad de análisis para grandes volúmenes de datos, que busca obtener pautas o patrones interesantes o significativos de acuerdo a planteamientos u objetivos planteados.

Y con respecto al $P 2 P$, por sus siglas en inglés peer to peer net, se trata de una red de ordenadores en la que todos o algunos aspectos funcionan sin clientes ni servidores fijos, sino a través de una serie de nodos que se comportan como iguales entre sí. Las redes $P 2 P$ permiten el intercambio directo de información, en cualquier formato, entre los ordenadores conectados, lo que ha suscitado gran polémica entre defensores y detractores de las leyes de derechos de autor. Napster, introducida en internet por Fanning, fue el comienzo de las redes $P 2 P$.

¿Factor común entre estos términos: Big Data, Data Mining y P2P? Varios, pero el que vamos a resaltar es que, todos y cada uno de esos procedimientos trabajan con lenguaje digital: representan el mundo, las experiencias, las palabras y las cosas traducidas a números. En efecto, tanto los Big Data como la Data Mining encuentran su razón de ser en los datos, enormes cantidades de datos. Y ¿en qué consisten esos datos? pueden consistir en muy diferentes cosas, procesos, acciones, experiencias, etc., pero siempre van a consistir en el registro digital que para cada uno de ellos se haga en la interfaz, que los torna numerables, calculables, manipulables, modelables, predictibles. Una vez que ha sido neutralizada en ellos toda remisión a realidades mostrencas y subjetivas, analógicas, una vez, en fin, que sucumban a la estandarización digital, entonces se va a tratar siempre ya de trasiego de señales, de transferencia de mensajes, de flujos de información. Esto, para empezar debiera dar elocuencia a nuestro aserto de que estamos siendo testigos de un secuestro redoblado a la autonomía, social e individual, en esta época del auge de los Big Data y las Data Mining.

Ni qué decir de las redes sociales. Como si las prácticas de sociabilidad ahora se verificaran a través del teléfono móvil, la tableta o el ordenador (en realidad es el teléfono móvil el que comanda los intercambios). En uno de los grupos de discusión que trabajamos, hizo falta intervenir tres veces (desde el lugar del preceptor) para inquirir al grupo respecto si actualmente no había lugares y maneras de sociabilizar presencialmente. Tal era la polarización del habla grupal en torno a los tópicos de internet, Facebook, Whatsapp o Tinder. Y en otro, una de las intervenciones fue con 
la pregunta “ ¿y cómo es la sociabilidad cuando los jóvenes están juntos, reunidos, presentes unos y otras?"

Constatamos que las redes sociales son una de las "vetas" más importantes y valiosas de donde se produce/extrae esa información, ese ingente volumen de datos. Pues como dicen Pérez y Gardey (2013):

Con la llegada de la Web 2.0 se produjo un fenómeno social que cambió para siempre nuestra relación con la información, principalmente porque nos hizo parte de ella: en la actualidad, una noticia acerca de una manifestación en contra del maltrato animal no está completa sin mostrar cuántos usuarios de Facebook leyeron y disfrutaron de la misma, qué porcentaje de lectores está a favor del movimiento, y los comentarios, que muchas veces aportan datos importantes o señalan errores. (Pérez y Gardey, 2013, s.p.)

Los datos recogidos en México por el INEGI ${ }^{3}$ (2016) muestran la clara predominancia del recurso a dispositivos electrónicos aptos para estar conectados a las redes.

Imagen 1. Usuarios de celular inteligente, según conectividad a internet y tipo de conexión, 2016 (\%). Fuente: INEGI, ENDUTIH, 2016.

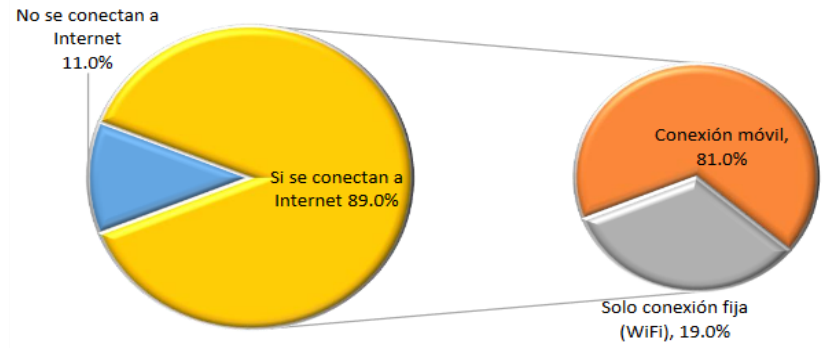

Las redes sociales se revelan, también, como redes de pescar, si observamos que a través de ellas las personas dejamos rastros de cómo nos presentamos, a quiénes saludamos, con quiénes nos relacionamos y en qué términos. Huellas de los ámbitos, objetos, lugares y temas que nos interesan, los problemas que nos interpelan, las dificultades que nos agobian. Huellas de nuestras aficiones y gustos, así como de nuestras aflicciones y nuestras fobias, etc. Y todo esto no sólo en un momento acotado, sino que el rastro es también cronológico, basta con modelarlo longitudinalmente para ver nuestras trayectorias: como consumidores, profesionistas, trabajadores, buscadores de pareja, o de aventura, o de sexo, ociosos flâneurs de la red, etc. Entonces no podemos dejar de preguntarnos ¿cómo es posible que las personas, notoriamente en mayor proporción las más

3 Instituto Nacional de Estadística y Geografía, 
jóvenes de 40 años, no muestren apenas reparos o no muestren ninguno en absoluto frente a este descarado acecho y registro de nuestros pasos? En el caso de México es muy clara la brecha generacional en cuanto al uso de internet como se aprecia en la siguiente gráfica, distribuyéndose la amplia mayoría de ellos en un rango de 12 a 34 años.

Imagen 2. Usuarios de internet por grupo de edad, 2016 (\%).

Fuente: INEGI, ENDUTIH, 2016.

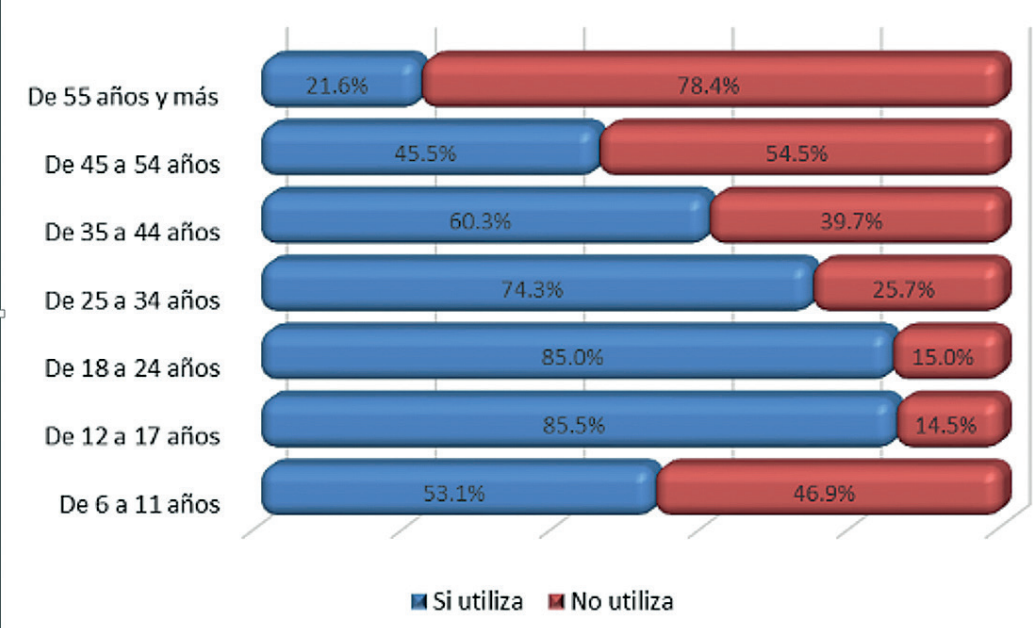

Lo que se ve y se escucha de las redes sociales es para algunos, y quizá notoriamente en mayor proporción entre los mayores de 50, desconcertante, o al menos inquietante. Pues en las redes sociales se ha venido montando una suerte de otro nivel de la existencia porque hay textos escritos, en audio, música, imágenes en los que la gente entrega y exhibe información sobre sus vidas, opiniones, habilidades, gustos.

Desde esa estandarización en lo digital, que consiste en traducir todo a números, el mundo y las cosas y la vida parecen perder sus cualidades. Todo se estandariza, todo se convierte en combinaciones de ceros y unos (lenguaje binario).

Y pensamos que, en parte, esto está promoviendo el efecto consecuente de vigorizar una mística de la cantidad en detrimento de la cualidad. Así, ahora lo valorado se apoya en números — número de eventos, número de visitas, número de "me gusta"y ante la falta o descrédito de la calidad, la autoestima de las personas se alimenta de popularidad. Se busca entonces la aceptación.

Pero pongámonos en un poco grato escenario de que las condiciones actuales de nuestras sociedades fuesen a más, condicionando la existencia social al hecho de estar bien ubicados en la Web 2.0, a mostrarse y discurrir en las redes sociales. En ese caso supuesto, habrá grupos sociales y regiones del mundo con menos existencia social, pues algo conocemos de la capacidad instalada de tecnología de la información y comunicación y de su distribución y accesibilidad en el mundo. 
Imagen 3. Tasa de penetración de internet global de 2009 a 2017 por región.

Fuente: Statista. The statistics portal.

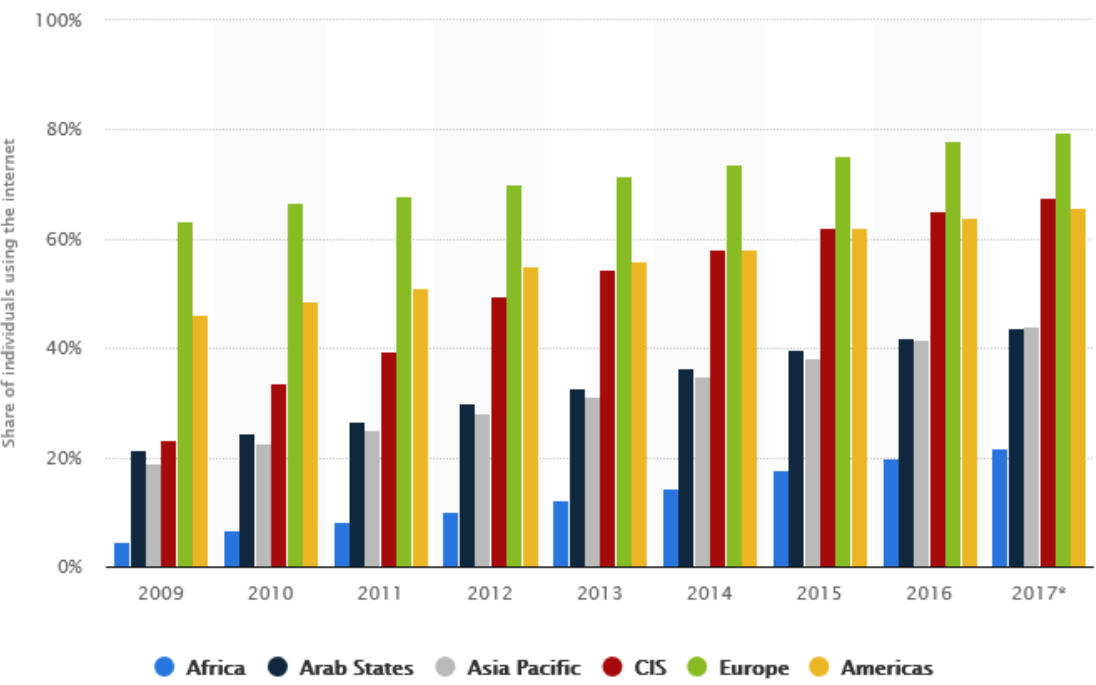

Al fragor de este contexto Pentland, profesor del MIT, declara:

Los recientes avances en comunicación inalámbrica y sensores digitales han hecho posible la observación del comportamiento humano natural y cotidiano con una minuciosidad previamente inalcanzable. Esto ha dado como resultado revolucionarias herramientas de medición, como el ya mencionado sociómetro, que nos proporciona una perspectiva «con ojos de Dios» de nosotros mismos. Por vez primera podemos seguir con precisión el comportamiento de gran cantidad de personas mientras desarrollan habitualmente sus vidas. (Pentland, 2010, p. 13)

Entretanto algunas empresas han devenido grandes o importantes desde el momento en que nos venden los instrumentos/aparatos para conectarnos, "comunicarnos", exhibirnos, en definitiva para existir en internet. Otras venden el servicio de hacer eso posible, toda vez que ya tenemos el instrumento. Otras se encargan de registrar las huellas que dejamos en nuestras andanzas por las "redes sociales" y demás aplicaciones desde nuestros gadgets.

Consigna Marvakis (2017, s.p.):

¿Qué es eso que vibra, parpadea y nos llama? Ya en el año 2014 Byung-Chul Han lo llamaba Big Data, y lo relacionaba con otra cuestión: el agobio con el cual inundamos las redes con información indiscriminada sobre nosotros, de manera tal que el concepto de "protección de datos" se vuelve obsoleto.

Y aún otros más, como puede deducirse, venden el servicio de procesamiento y sistematización de esos grandes números, y esos volúmenes de datos. Al respecto de las dimensiones que alcanzan esos volúmenes de datos, se nos dice que el universo 
digital duplica su extensión cada dos años, y que si en 2013 su tamaño era de 4.4 zettabytes, para 2020 alcanzará los 44 zettabytes. Y cuando estas expresiones de medida se traducen a ejemplos en pos de la elocuencia, en los discursos de actores o entidades concernidas con el desarrollo de las tecnologías digitales, vemos entonces la escala de la desmesura como se presenta en el estudio de EMC sobre el universo digital "Si el universo digital estuviera representado por la memoria en una pila de tabletas, en 2013 se habrían alcanzado dos tercios de la distancia hacia la luna. Para 2020, habrá 6.6 pilas desde la tierra hasta la luna." (EMC, 2014).

Esta cita representa una expresión típica y, temiblemente puede que también una expectativa, a barlovento con el imaginario de nuestra época y con la inercia de los afanes y las instituciones: sociedades con aviones supersónicos, con trenes de alta velocidad, "Citius, altius, fortius".

La sonoridad de estos discursos en buena medida se sostiene en el campo de la publicidad, y para el caso el siguiente ejemplo de texto publicitario:

Procesadores "Extreme Edition". Entre ellos nos encontramos a lo que podemos considerar como el buque insignia de la terna, el Intel Core i7-6950X. ¿Qué lo hace especial? Pues muchas cosas, pero principalmente la presencia de diez núcleos en su CPU.

En el mundo de los ordenadores de escritorio la carrera por la velocidad de ciclo quedó a un lado para dejar paso a los núcleos, y aunque en 2011 ya ofrecieron procesadores Xeon con esa cantidad de núcleos, no lo habíamos visto en la oferta doméstica hasta ahora. El salto económico también es llamativo, ya que por él nos van a pedir $\mathbf{1 . 7 2 3}$ dólares.

El nuevo procesador podrá correr a $3 \mathrm{GHz}$, y tendrá modos de funcionamiento "Turbo Boost 3.0" que lo lleven hasta los 3.5GHz. Es capaz de ejecutar hasta 20 hilos de trabajo de forma simultánea, todo un monstruo para trabajar con múltiples tareas. (Puerto, 2016, s.p.)

Los jóvenes y los niños de hoy crecen en una sociedad que produce millones de dispositivos multitareas y que incuba sueños de la mujer y el hombre multifuncionales. Aquí encontramos un ejemplo paradigmático del cómo la sociabilidad, como la estamos entendiendo, es puesta en predicamento. Cuando hablamos de un vector de componente centrífugo, lo que queremos es llamar la atención sobre un entorpecimiento del trato social, de la sociabilidad en general, de sus formas, de sus ritmos y sus fórmulas. Y proponemos la idea de vector centrífugo haciendo eco, en un sentido leve, de la teoría de campo de Kurt Lewin. Vector centrífugo: el que describe la fuerza que, en un sistema rotatorio, expele a los cuerpos en la dirección opuesta al eje del mismo. Si pensamos a la sociabilidad como las formas de concurrencia intersubjetiva y trato social, creemos que no resiste muchas pruebas para su demostración el hecho de que las nuevas tecnologías - y fundamentalmente las posibilidades que se abren con la Web 2.0 - alteran, obstaculizan o enrarecen la sociabilidad. Pues compartimos con Vázquez que "La complejidad del ser humano se disgrega en un contacto instrumental que rehúye la franquicia del cara a cara" (Vázquez, 2014, s.p.).

$\mathrm{Y}$ es que somos un cuerpo dotado de cinco sentidos, los cuales siempre trabajan/ intervienen orgánicamente, conjuntamente, en la "decodificación" de la circunstancia en la que nos encontramos. No podemos ir a algún lugar sin nuestros sentidos 
o, por decir, sólo con vista y olfato, dejando a resguardo en casa el oído, tacto y gusto. Ahí donde nos encontremos estamos con los cinco sentidos. Y es bueno que así sea pues es gracias a ellos que conseguimos un primer nivel de decodificación de la circunstancia, necesario para orientarnos, saber estar, proceder o discurrir. Y hablo de un primer nivel porque, el animal humano potencia, articula y organiza la información que sus sentidos le reportan de un modo particular y determinante de sus comportamientos e igualmente de sus actos. Pues los seres humanos no sólo nos comportamos sino que actuamos.

Una manera de exponerlo sería: es por el concurso de todos nuestros sentidos, pero iluminados, además, por nuestra potencia reflexiva, por lo que se produce la inteligencia de la situación, de nuestra situación. Y la situación es siempre aquí/ahora. Así, desde el momento en que la presencia física es el salvoconducto de nuestra inteligencia (intus-legere: leer desde dentro) para llegar a entender, a comprender, a dar sentido a la circunstancia y a la situación, nada puede sustituir cabalmente - sin pérdida - la experiencia presencial, el estar ahí/en ese momento. Somos una presencia, un cuerpo que ocupa sitio en el espacio, en el mundo, y que mientras somos (mientras estamos, mientras existimos) es de nuestro estar-ahí-en ese momento de donde hacemos la experiencia de las cosas. Vía lectura de la situación, de la circunstancia, de las relaciones, los procesos, las actividades, etc., inteligimos. Es así como conocemos. Estamos viendo que las tecnologías emergentes generan las condiciones prácticas para provocar condicionamientos "multitarea". La modalidad multitarea, pues, disminuye nuestra presencia, provoca una mengua, un menoscabo de nuestro estar-ahí-en ese momento.

La multitarea, como el barullo de distracciones constantes, disminuye nuestro estar-ser-ahí-en ese momento. Porque nuestra atención se ve subdividida, distribuida en dos o tres canales (o en más) en un lapso de tiempo. ¿Cómo poder estar/ser, ya no en varios canales en los que se prodiga nuestra atención, sino en el ahí-ese-momento de nuestra situación presencial, ahí donde estamos en tanto somos cuerpo, con nuestros cinco sentidos? ¿Cómo vamos a hacer lectura de nuestra situación?, ¿y cómo se hace la lectura de los otros focos de atención?

Las nuevas tecnologías, al generar las condiciones multitarea, propician de cara a la sociabilidad la construcción de algo así como un campo minado. Con la enorme diferencia de que aquí las minas no hacen explotar a la gente, sólo la "sacan de la jugada" de la sociabilidad, la distraen, la dividen, la confunden.

Provocan, pues, una sociabilidad también disminuida, la empobrecen al sabotear, y por tanto disminuir, el estar-ahí-en ese momento de las personas por entero. Cada uno de los intervinientes está y a ratos no está, porque debe hacer lectura y hacerse cargo de los otros dos o tres focos de atención a través de otros canales de comunicación.

\section{Estrecho cerco a la autonomía}

Mandosio, citado por Cul de sac (2011, p. 6) plantea:

Un sistema técnico no es nunca exclusivamente técnico, sino igualmente económico, social, y político, pues se entiende que la interdependencia de las técnicas en el seno de un sistema dado se inscribe a sí misma en un conjunto de relaciones 
económicas, sociales y políticas [...] No es jamás neutro [...] Los individuos que coexisten, en una sociedad dada, no se encuentran jamás en una situación de elección abierta, sino que son determinados en mayor o menor medida. La autonomía absoluta no existe, sea en relación con la técnica o con cualquier cosa: es una proyección intelectual. Existen, por el contrario, sistemas técnicos (y, por tanto, indisociablemente, económicos, sociales, políticos) que dejan más autonomía a los individuos que otros sistemas. La pérdida de autonomía que ha representado el maquinismo es incontestable.

Resulta irónico que en unas sociedades en las que parece verse ilustrado de manera más acabada lo que Marx pensaba con su idea de un "general intellect", destaque un efecto centrífugo que nos separa, expulsa, y dispersa. Por ejemplo, ¿cuántos aparatos de teléfono de cable habría en el mundo todavía a mediados de los años 1990? Sabiendo que no había un aparato por familia, al menos en la mayor parte del mundo, que es la más pobre. Se trataba, pues, de aparatos que compartían las familias de clase media y alta y que compartía la colectividad en las casetas públicas. En el año 2000, según el sitio especializado en tecnología de Clarín (2015), la UIT ${ }^{4}$ de Naciones Unidas calculó en 700 millones el número de teléfonos móviles, Pero ahora parece haber un smartphone por cada persona, pues para 2015, esa cifra se había elevado a 7,000 millones, casi el número de la población mundial.

Pensamos que en esto se revela ese nuevo cerco a la autonomía, en la línea de lo que plantea Mandosio respecto a la pérdida de autonomía que ha supuesto el maquinismo. Pero lo que estamos presenciando ahora hace blanco ya de una manera sofisticada en los individuos. La primera ola de industrialización produjo máquinas que se insertaron en los talleres y fábricas, y eran de responsabilidad y atención colectiva, independientemente del régimen de propiedad privada en el que aparecen y proliferan. En la segunda revolución industrial ocurre lo mismo, pero con máquinas y aparatos perfeccionados, y algunos de ellos por su diseño y funcionalidad son ubicables en domicilios o en clubes, pues de esta segunda ola o revolución industrial son producto el cinematógrafo, el teléfono y fonógrafo, el automóvil; además también, se dice, es la revolución de la electrificación. Pero ahora, en esta tercera revolución industrial, científico tecnológica o de la inteligencia como la caracteriza Riffkin (2011), se da una convergencia de aprovechamiento de energías renovables con las nuevas tecnologías de la comunicación. Lo que encontramos es que los nuevos insumos y productos tecnológicos son en mucho de uso individual: ordenadores personales y portátiles, tabletas, teléfonos "inteligentes". Así proponemos considerar dos ejes de avance del maquinismo: 1) avanza en extensión y ahora incide en cada individuo, al menos los tiene por objetivo; y 2) avanza en intención, pues con esa logística de consumo y uso de los aparatos dependemos de más proveedores de servicios: el fabricante y primer vendedor del dispositivo, que constantemente nos estará ofreciendo mejoras y nuevas funcionalidades; una gama de competidores que ofrecen sus versiones de programas; proveedores de internet; proveedores de aplicaciones y de plataformas, etc.

Y ¿qué ha generado que en un lapso de veinticinco o a lo sumo treinta años de "nuevas tecnologías" en nuestras sociedades, se hayan alcanzado (y no se avizora aún

4 Unión Internacional de Telecomunicaciones, Organismo de Naciones Unidas. 
el techo) las cotas de desmesura que son las actuales? Winner (1985) distingue dos tipos de explicación frente a una cuestión como ésta. Estaría la explicación desde un determinismo tecnológico ingenuo, "la Idea de que la tecnología se desarrolla únicamente como resultado de su dinámica interna y, entonces al no hallarse mediatizada por ninguna otra influencia, moldea la sociedad para adecuarla a sus patrones". En segundo lugar estaría la explicación desde una posición que vamos a llamar determinismo social de la tecnología, "las tecnologías son el resultado de movimientos e intereses de fuerzas sociales y sobre todo económicas" (Winner, 1985). Pero pensamos, junto con este profesor del Rensselaer Polytechnic Institute en Troy, New York, que no debemos quedarnos satisfechos con la explicación del determinismo social de la tecnología. Como él dice, no conformarnos con sólo haber descubierto "la mano de los poderosos tras un determinado ejemplo de cambio tecnológico". Porque con respecto a las tecnologías de la información y la comunicación —y en conjunto a las llamadas tecnologías emergentes - no es sólo que su diseño, construcción y puesta en funcionamiento - a través de un impresionante despliegue productivista y comercial - obedezca a una condición de la co-relación de fuerzas políticas y económicas en nuestras sociedades. Todo esto es cierto, pero lo que está diciendo Winner es que, además debemos fijarnos en las propias características de los objetos técnicos/ tecnológicos, e interpretar los significados que esas características portan.

Las características físicas y funcionales de los objetos: sus formas, su color, sus diseños, sus emblemas y logos. En esta línea no deja de resultar llamativa la siguiente observación de Franklin (2013, p. 22):

\begin{abstract}
What is relevant here is to note how critiques of the internet and its cyberspaces as democratizing, namely, empowering in principle (...) are most concerned with the historical conjuncture of neoliberal ideology, postmodernity, consumerism, and the rise of the internet as mass media on a global scale.
\end{abstract}

No terminamos de entender cómo pueda hacerse una reflexión crítica y productiva respecto no sólo a las nuevas tecnologías, sino a cualquier tecnología que se implanta y difunde socialmente, si no se dispone de un enfoque que contemple al objeto en su engarce con las fuerzas y factores sociales por los cuales y con los cuales ese desarrollo tecnológico existe: en la producción, la legitimación, las prácticas sociales.

Pero volviendo a lo señalado por Winner (1985) respecto a los objetos técnicos, un rubro ineludible es el de sus utilidades. Los usos y prácticas que esos dispositivos promueven entre los usuarios, alteran y modifican los escenarios y modos de los encuentros, inauguran y facilitan otras vías y maneras para la comunicación. Los significados que todo esto desata y conlleva, revierten, por supuesto, en los ámbitos de la sociabilidad, e intervienen en la producción del sentido en los escenarios de la vida cotidiana.

En una importante medida son precisamente las características físicas y funcionales de las herramientas y aparatos del almacenamiento, procesamiento y trasiego de información las que han habilitado un potente "vector centrífugo" en el "campo" de la sociabilidad. La paradoja es solo aparente, si pensamos con Boltanski y Chiapello (2002) que lo que se perfiló desde los años 1980 y siguientes es un neocapitalismo conexionista e individualizador. En este trabajo hemos querido comenzar a señalar 
uno de sus efectos múltiples: la re-composición de la circunstancia y las dinámicas de la interacción social, una re-disposición de las cosas que se anuncia incisiva para la sociabilidad. Desde el arriesgado desdibujamiento de las fronteras entre "lo público" y "lo privado", que sin embargo se manifiesta de diferente manera, por supuesto, según se trate de "propiedad privada" o de "vida privada". Hasta el no menos riesgoso borramiento de la diferencia entre ciudadano y consumidor. Y es que desde su realidad de objetos en una sociedad de mercado, los gadgets no pueden no hacer de señuelos, pues gozan del misterioso y teológico brillo de la mercancía, Ya al comprarlos hemos caído en las redes, hemos sido pescados. Ahora bien, son un tipo particular de mercancías, se ofrecen siendo insignias del ocio, la distracción, de la holgura del tiempo libre, es decir del reposo. Y también de la libertad, pues, se nos dice "nos dan independencia", "aumentan nuestra autonomía", "multiplican nuestras capacidades" "incrementan nuestras posibilidades". Cierto, pero lo hacen en distintos sentidos, o mejor, como lo muestran los resultados parciales de nuestras pesquisas, conllevan múltiples y diversas consecuencias. Nosotros nos enfocamos a destacar las que ponen en causa a la sociabilidad. En este sentido, las nuevas tecnologías nos permiten hablar con alguien que está en Malasia, ver su imagen, recibir las fotografías que ha tomado en su viaje, las de apenas hace tres minutos, allá, a 20 mil kilómetros de distancia de nosotros. Además permiten estar monitoreados en todo momento por la empresa o la dependencia para la que trabajamos. Y también abonan al reblandecimiento de los límites entre tiempo de trabajo/producción y el tiempo libre. Cada uno de estos casos tiene implicaciones particulares para la sociabilidad, tal y como la estamos concibiendo. Por ejemplo, como dice Andreas Wittel: "La asimilación del trabajo con el juego se corresponde con el borramiento de los límites entre trabajo y vida privada, entre colegas y amigos y entre colegas y clientes" (Wittel, 2001, p. 69).

Un entreveramiento de juego-diversión-aprendizaje-producción está a la orden del día, facilitado por las nuevas tecnologías; estas se muestran como factores coadyuvantes a la infantilización de ethos en las sociedades contemporáneas, para decirlo como Barber (2008). Se hacen reuniones de trabajo en pubs, se perfilan proyectos y se toman decisiones en fiestas.

"El trabajo tiene que ser divertido ¿por qué preocuparse de trabajar duro si no es divertido?" Esta es una frase frecuentemente escuchada entre la gente de los new media. Esta actitud juguetona conduce a una intensificación del trabajo. Escuchan música mientras trabajan, usan drogas mientras trabajan, invierten un buen tanto de sus horas laborales en cafés y pubs. Y el propósito de esta integración de lo lúdico con el trabajo es una mejor ejecución. (Wittel, 2001, p. 68)

Desde el otro costado podemos encontrar que, a su vez, las aficiones, los entretenimientos y actividades de esparcimiento pueden estar intervenidos con registros, mediciones y evaluaciones. Si la sociedad del conocimiento es una sociedad digital, numérica, pues habrá qué medirlo y calcularlo todo. Como señala el Comité invisible: "hoy tenemos algo que ya es directamente observable: el funcionamiento del sensor más polivalente de todos: yo. Hice footing e, inmediatamente, compartí mi ruta, mi tiempo, mi rendimiento y la evaluación de mi carrera" (Comité invisible 2015, p. 43). 
Eso de ser rehén del mercado, como mencionamos en la introducción de este escrito, es una condición que la sociedad ya conoce, si acordamos con Polanyi (2017) que desde finales del siglo XIX con la consolidación del modelo del liberalismo económico la sociedad quedó a la zaga de la economía. Desde entonces las condiciones de posibilidad para el ser social quedaron subtendidas a la forma "mercado". Cierto, pero ahora, con los pistas que hemos recogido en cuanto a los éxitos de ventas, de usos y júbilos con los gadgets, las reality mining, los zettabytes etc. y las que recogemos de Mandosio y de Winner, lo que estamos avizorando es ese otro nivel de encantamiento y correlativa pérdida de autonomía. Diremos con Arnaud Tomès, se trata de una de las claves de "«la servidumbre voluntaria» del hombre contemporáneo con respecto a los objetos que produce" (Tomès, 2015, p. 104). Clave que puede ayudar a disipar el enigma de lo que con oportunidad ha consignado César Rendueles,

una realidad fascinante del capitalismo es que se ha impuesto a escala mundial a pesar de carecer de grandes discursos de legitimación. La sociedad de mercado no tiene ningún Pericles, Catón o San Agustín. No hay declaraciones de derechos, actas fundacionales ni monumentos. Es llamativo porque pocas sociedades han exigido una lealtad tan heroica y una ritualización tan extrema de los comportamientos cotidianos. (Rendueles, 2013, p. 19)

¿Cómo encajar, cómo digerir esa aquiescencia al capitalismo por parte de tantas sociedades y de los individuos de esas sociedades? ¿Cómo?, cuando con Castoriadis también nos preguntamos “¿Por cuánto tiempo esta humanidad seguirá obsesionada por esas inanidades y esas ilusiones que se llaman mercancías?” (Castoriadis, 1996, p. 96). Pero es que el mismo Castoriadis (1999) se encarga de mostrarnos el espesor de las cosas cuando en otro trabajo, La rationalité du capitalisme, criticando la presunta racionalidad de este sistema, señala que la única justificación algo legítima del capitalismo es la que dio Schumpeter en su libro Capitalismo, socialismo, democracia y que resume así: "Ciertamente el sistema es cruel, injusto y turbulento, pero él provee la mercancía, y deje de protestar que esta es la mercancía que usted quiere" (Castoriadis, 1999, p. 87).

Somos rehenes, también, de la propia materialidad y funcionalidad de nuestros dispositivos y nuestros gadgets, de nuestros entornos codificados y automatizados.

En efecto, hoy como nunca antes la gente está conectada, hablando por sus portátiles, aún mientras camina, conduce, o viaja en bicicleta. Escribiendo, leyendo mensajes, gigs, etc., mientras camina, va en el metro, trabaja, come con sus hijos, pasea con su novio, etc. En conjunto, esto forma toda una malla de flujos, de datos, de información; acaso valga la imagen como una instantánea de la "sociedad red". Pero lo que estamos planteando, y vamos a seguir explorando, es la hipótesis de la activación de un vector de componente centrífugo en el campo de la sociabilidad. Son elocuentes también con nuestra hipótesis los rasgos de la network sociality consignados por Wittel (2001): esa socialidad red del neocapitalismo conexionista no supone "pertenencia" sino "integración-desintegración"; la vía principal de la relación no es "narracional" (caracterizada por la duración) sino "informacional" (caracterizada por su efimeralidad). Las relaciones, pues, son fugaces y transitorias, a veces de encuentros intensos pero efímeros. Las valencias de las relaciones se combinan y confunden entre trabajo y juego. Su plataforma operativa y funcional fundamental 
es la tecnología de la comunicación y el transporte. También Rendueles señala que aunque el actual capitalismo pueda ofrecer una sociabilidad abundante, esta es, no obstante, "extremadamente epidérmica": "El capitalismo es compatible con las relaciones sociales reticulares y cierto nivel de cooperación trivial, no así con el cuidado mutuo" (Rendueles, 2013, p. 148).

\section{Concluyendo, por ahora}

Hemos empezado a formular una hipótesis según la cual«en la sociedad del capitalismo conexionista del siglo XXI la plataforma instalada de las tecnologías de la comunicación y el transporte activan un vector de componente centrífugo para el "campo" de la sociabilidad». Pensamos que esta idea conlleva un doble desafío de cara a un ejercicio de la autonomía, en un sentido radical, por parte de la colectividad y los individuos. Por un lado, está el desafío del encanto o fascinación propia de la mercancía, puesto que "el consumo de mercancías" en el actual campo de lo posible constituye - a pesar de todo - un modo de ejercer la libertad, un modo de elegir, de optar, de existir como ser social. Porque sabemos que este ser social que somos puede pasar diez horas al día trabajando, y así cinco o seis días por semana, y que cuando obtiene su pago puede decidir comprarse el nuevo modelo de, algo. Así es como puede devenir "un ser social de su sociedad", y tener garantizada su inclusión, su integración, al tener asegurado el reconocimiento de sus iguales, de los de su clase, de su familia, de su gremio, de su barrio. Y también de sus proveedores/vendedores. Pero aquí se nos presenta otra incógnita, sobre las posibilidades tácticas y estratégicas que las nuevas tecnologías abren de intervención y moldeamiento insidioso del trato social. Como refiere Marvakis (2017, s.p.), en su lectura de Byung —Chul Han:

El paso psicopolítico, sin embargo, está en el giro -que entre 2014 y 2017 podemos corroborar sin esfuerzo- entre la vigilancia pasiva y el control activo de esa información. ¿Y si el poder fuera entonces capaz de intervenir en nuestra mente y condicionarla a un nivel prerreflexivo? Al fin y al cabo, basta estudiar un poco lo que nos gusta, lo que compartimos y lo que comentamos en Facebook hace apenas un rato para anticipar con bastante éxito lo que nos va a gustar, lo que vamos a compartir y lo que vamos a comentar un rato más adelante. En este punto, por supuesto, conviene pensar más allá de Facebook y concentrarnos en internet en general, es decir, concentrarnos en la noción de una plataforma digital a la que está conectada casi la mitad de la humanidad.

Pensamos que la teoría del reconocimiento de Honneth $(2000 ; 2006)$ tendrá mucho que aportarnos para proseguir esta elucidación.

A ese nivel del ordenamiento encantado de las mercancías se agrega ahora, y aquí topamos el segundo desafío, toda esta parafernalia de las nuevas tecnologías, que, constituyendo también mercancías, lo son con una funcionalidad incorporada, con una operatividad que viene a trasponer las oportunidades y escenarios del trato social. Se está, de hecho, operando una re-disposición de las cosas para el despliegue de la sociabilidad. Hay un vector centrífugo que nos aleja, nos encierra y peligrosamente parece que nos inhibe e inhabilita para el trato y la correspondencia social 
al poner verdaderos obstáculos, por ejemplo, a la experiencia de la empatía (Turkle, 2017). Porque, lo formularemos así, por un lado ahora nosotros, las personas, vivimos bajo condiciones de una sociabilidad intervenida, somos parte del campo de disputa de una colección de solícitos vendedores de muy variadas mercancías y de entre ellas las de proveedores de servicios de esa plataforma de la comunicación. De modo que en el decurso de nuestras navegaciones, y puede ser que entre una y otra llamada o intercambio de textos, o en los segundos que transcurren en lo que se establece la conexión e iniciamos una videoconferencia, nos aparezcan ofertas, sugestiones y demás mensajes que, obviamente, se interponen en el curso de la actividad en que nos encontramos o a la que nos disponemos. No podemos dejar de evocar el paralelismo entre lo que ocurre en las guerras con dos ejércitos beligerantes (cuando la confrontación causa víctimas y daños a la población civil) y el estado en el que nos encontramos en tanto que usuarios cotidianos de las plataformas digitales. En el proceso de comunicación, bien con uno mismo, bien con los otros, (en cualquier caso comunicación con los otros, sólo que directa o indirecta) nos hallamos frecuentemente interrumpidos, distraídos. Como si en el curso de nuestras conversaciones, que pueden ser con nosotros mismos —el pensamiento es un diálogo silente, dijo Platón-, debiésemos acostumbrarnos a desempeñarnos en medio del barullo provocado por una segunda línea de comunicación que se nos impone y que es aleatoria, aparece cuando "ella" — el otro de esos mensajes - quiere, para decirnos cosas como: a) que la licencia del antivirus está por vencer en siete días; b) que si queremos programar la hora de instalación y reinicio de nuestro dispositivo para la última actualización; c) que si queremos pasarnos a la nueva versión de la aplicación "x"; d) que el espacio en nuestra bandeja o en nuestra nube se ha reducido fuertemente, etc. Además de la variedad de anuncios y ofertas relativos a nuestras búsquedas o navegaciones recientes. De modo que nos vemos constreñidos a conversar y pensar, mientras usamos nuestro ordenador o nuestra tableta, bajo condiciones de asedio. Como enuncia Franklin (2013) hemos pasado de una "battle of the browsers" a una "battle of the search engines" (p. 65-92).

¿Para qué poner en común si podemos ver lo mismo cada quien en su pantalla? ¿Qué estamos gastando seis veces más de: batería, dispositivo mismo, milivatios y o decibelios? Bueno oye, sólo es un juego ${ }^{5}$. Este diálogo imaginado quiere ilustrar el vector centrífugo que para la sociabilidad es propiciado con las condiciones de la capacidad instalada de las tecnologías emergentes, particularmente la plataforma y los dispositivos digitales. Un aspecto importante a considerar es el de ¿qué tanto este orden y dinámica de la interacción social puede llegar a banalizar y, acaso corroer, el trato social? Pues, poco a poco, las condiciones de lo digital facilitan la evitación del contacto, de los encuentros cara a cara, de largas reuniones para la toma de acuerdos, del aprendizaje de campo en la negociación de las reglas del juego con los amigos. Sherer lo expresa de un modo que nos viene muy bien citar aquí: " $L$ ” analogique a un coût physique qui favorise la rareté, le numérique a un cô̂t marginal quasiment nul, qui favorise l"abondance et l"ubiquité"" (Sherer, citado en Fabre, 2017, p. 30 ${ }^{6}$. Mientras la planta física-técnica que hace posible el funcionamiento de la Web 2.0, digamos mientras el Hyperhardware se mantenga funcionando, mientras

\footnotetext{
Resaltado por los autores.

"Lo analógico tiene un costo físico que favorece la rareza, lo digital tiene un costo marginal prácticamente nulo que favorece la abundancia y la ubicuidad" (traducción propia).
} 
en nuestras ciudades continúe la oferta y provision de $p c$ "s, lap tops, smartphones, tablets, baterías, cables, etc., en suma, mientras se mantengan las condiciones para facilitar que la gente pueda evitar ir a las cosas y los lugares y, muy particularmente, ir al encuentro presencial con el otro, en esa medida encontramos que la sociabilidad estará sitiada. Señala Turkle (2017) que nuestros dispositivos electrónicos le ofrecen a nuestro cerebro un menú continuo, e infinitamente entretenido de estímulos, que requiere menos esfuerzo, entonces "abandonamos el camino lento, en el que hay que esperar, escuchar y dejar que la mente repase las cosas. Nos apartamos del ritmo de la conversación humana" (Turkle, 2017, p. 56). Efectos parecidos, nos dice la misma Turkle (2017) han sido identificados por el psicólogo cognitivo de Stanford, Clifford Nass y un grupo de colaboradores (Pea et. al., 2012) sobre el desarrollo emocional de jóvenes. Nass encontró que las jóvenes que se calificaban de «muy conectadas» tenían ostensiblemente menos capacidad para identificar sentimientos en otras personas, e inclusive en ellas mismas y además se sentían menos aceptadas por sus iguales que aquellas que usaban menos las redes sociales (Turkle, 2017). Podemos convenir que será porque esta abundancia y ubicuidad facilitadas por la plataforma digital, para, supuestamente "estar en cualquier lugar en cualquier momento, en realidad pueda ser "en cualquier momento", pero no "en cualquier lugar"; porque somos presencia, forma, tenemos un costo físico, no somos números, no somos dígitos con su casi nulo costo marginal. Si acaso, a través de la tecnología digital, podemos asomarnos a representaciones de los lugares; inclusive a la representación social con algún otra/o. En esta línea por ejemplo, recogemos de Harper la llamativa estimación de que la utilización de Skype constituye el $40 \%$ de los minutos de telecomunicaciones trasnacionales (Harper, 2016) Pero por lo visto, aun contando con que nuestras conversaciones a larga distancia incluyen la imagen de las personas interlocutoras, eso no es suficiente para formar seres humanos pues como dice el mismo Nass (2012), citado por Turkle (2017), "La tecnología no nos ofrece una educación sentimental", a lo que Turkle añade (y nosotros con ella): "pero la gente sí" (Turkle, 2017, p. 58).

Mientras no extendamos el campo de los posibles, mientras no plantemos nuevos motivos en el campo de positividades y abramos nuevas rutas para las prácticas de reconocimiento no se insinuarán nuevos senderos para el goce. Y seguiremos instalados bajo el imperio de la estéril necedad del "time is money".

\section{Referencias}

Barber, B. (2008). Consumed. How markets corrupts children, infantilize adults and swallow citizens whole. New York: W.W. Norton Books.

Barranco, R. (2012). ¿Qué es Big Data? Todos formamos parte de ese gran crecimiento de datos, [en línea]. Disponible en https://www.ibm.com/developerworks/ssa/local/im/quees-big-data/

Boltanski, L. y Chiapello, È. (2002). El nuevo espiritu del capitalismo. Madrid: Akal.

Carr, N, G. (2008) Is Google making us stupid? The Atlantic july/august 2008, [en línea]. Disponible en https://www.theatlantic.com/magazine/archive/2008/07/is-google-making-usstupid/306868/

Castoriadis, C. (2007). La institución imaginaria de la sociedad. Buenos Aires: Tusquets editores.

Castoriadis, C. (1999). Figures du pensable. Les carrefours du laberynthe 6. Paris: Seuil. 
Castoriadis, C. (1996). La montée de l'insignificance. Les carrefours du laberynthe 4. Paris: Seuil.

Clarín-tecnología (2015). El mundo ya tiene la misma cantidad de celulares que de gente, [en línea]. Disponible en https://www.clarin.com/tecnologia/mundo-misma-cantidadcelulares-gente_0_BJchxmYwXg.html [2018, 20 de febrero].

Comité invisible (2015). Fuck off Google. En Tiqqun (2015). La hipótesis cibernética. Madrid: Acuarela y A Machado.

Corcuff, Ph. (2006). Individualité et contradictions du néocapitalisme. SociologieS. Theories et recherches, [en línea]. Disponible en http://sociologies.revues.org/document $462 . \mathrm{html}$ [2017, 12 de julio].

$\mathrm{Cul}$ de Sac (2011). El Acondicionamiento neotecnológico. Cul de Sac Revista de pensamiento crítico (2) p. 6.

Degenne, A. y Forsé, M. (1994). Les réseaux sociaux. Paris: Armand Colin.

EMC-IDC. (2014). The Digital Universe of Opportunities: Rich Data and the Increasing Value of the Internet of Things, [en línea]. Disponible en https://www.emc.com/leadership/ digital-universe/2014iview/index.htm

Fabre, R. (avec la collaboration de Quentin Messerschmidt et Margot Holvoet) (2017). Les nouveaux enjeux de la connaissance. Dynamiques nuériques de l'accès et du partage. London: ISTE Editions.

Fabri (dirs.) (2015). Autonomie ou barbarie. La démocratie radicale de Cornelius Castoriadis et ses défis contemporains. Neuvy-en - Champagne: Le passager clandestin.

Franklin, M.I. (2013). Digital dilemmas. Power, resistance, and the internet. New York: Oxford University Press.

González Bernaldo de Quirós, P. (2009). Sociabilité Urbaine. En Hypothèses 1 (12): 295-303. Gurvitch, G. (1963). La vocation actuelle de la sociologie. Paris: PUF t.I.

Harper, R. (2016). Explorations in the gramar of "being in touch": From Locke to Winch, from SMS to Skype. En J. Floyd, and J. E. Katz (editors). (2016). Philosophy of emerging media. Understanding, appreciation, application. New York: Oxford University Press.

Honneth, A. (2000). La lutte pour la reconnaissance. Paris: Les éditions du Cerf.

Honneth, A. (2006). La société du mépris.Paris: La Découverte.

Iglesias L-G. (2017). La sociabilidad, una puesta al día. En Revista Ciencia@UAQro, 10 (2): 62-76. Disponible en http://www.uaq.mx/investigacion/revista_ciencia@uaq/ ArchivosPDF/v10-n2/art5_numpagina.pdf [2018, 9 de enero].

INEGI (2016). Encuesta nacional sobre disponibilidad y uso de las tecnologías de la información en los hogares [enlínea]. Disponible en http:// www. inegi.org.mx/ saladeprensa / aproposito/ 2016/ internet2016_0.pdf [2017, 10 de diciembre].

Jaucourt, L. (1765). Sociabilité, en D. Diderot y J-R D"Alambert directeurs Encyclopédie ou Dictionnaire raisonné des sciences, des arts et des métiers, par une société de gens de lettres. Paris 1765, t. XV p. 250-251.

Mandosio, J-M. (2000). Après l"effondrement- notes sur l"utopie néotechnologique. Paris: Éditions de L”Encyclopédie des Nuisances.

Marvakis, N. (2017). Facebook y psicopolítica. RevistaPaco [en línea]. Disponible en https:// revistapaco.com/2017/11/24/facebook-y-psicopolitica/. [2017, 8 de diciembre].

Nass, C. (2012). Is Facebook stunting your child"s growth? Pacific Standard. Santa Barbara (CA): Miller-McCune Center for Research Media and Public Policy, [en línea]. Disponible en https:// psmag.com/social-justice/is-facebook-stunting-your-childs-growth-40577 [2018, 19 de febrero].

Pea, R. Nass, C. Mehuala, L. Rance, M. Kumar, A. Bamford, H. Nass, M. Simha, A. Stillerman, B. Yang, S. Zhou, M. (2012). Media Use, face-to-face communication, 
media multitasking,and social well-being among 8-to 12-year-old girls. Developmental Psychology 48 (2): 327-36.

Pentland, A. (2010). Señales Honestas, el lenguaje que gobierna al mundo. Barcelona: Editorial Milrazones.

Pérez, J. y Gardey, A. (2013). Definición de Web 2.0. [En línea]. Disponible en https:// definicion.de/web-2-0/ [2017, 10 de diciembre].

Pessoa, F. (2015). Sobre o fascismo, a Ditadura Militar e Salazar, en José Barreto editor (2015), Fernando Pessoa, sobre o Fascismo, a Ditadura Militar e Salazar. Lisboa: Tinta da China.

Polanyi, K. (2017). La gran transformación. Los orígenes políticos y económicos de nuestro tiempo. México, DF.: Fondo de Cultura Económica. [orig. 1944].

Puerto, K. (2016). El Core 77 más potente de Intel tiene 10 núcleos y cuesta 1.723 dólares, [en línea]. Disponible en https://www.xataka.com/ordenadores/quieres-saltar-a-los-dieznucleos-con-los-nuevos-procesadores-intel-prepara-1-723-dolares-para-la-joya-de-losextreme-edition

Rendueles, C. (2013). Sociofobia. El cambio político en la era de la utopía digital. Madrid: Capitán Swing.

Rifkin, J. (2011). La tercera revolución industrial. Cómo el poder lateral está transformando la energía, la economía y el mundo. Barcelona: Paidós.

Rivière, C-A. (2004). La spécificité française de la construction sociologique du concept de sociabilité. Réseaux $1 \mathrm{n}^{\circ} 123,207-23$.

Simmel, G. (1971). On individuality and social forms. Selected writings, edited by Donald N. Levine. Chicago: The University of Chicago Press.

Simmel, G. (1981). Sociologie et épistémologie. Paris: PUF.

Simmel, G. (2002). Cuestiones fundamentales de sociología. Barcelona: Gedisa.

Tomès, A. (2015). La fascination de la merchandise. En Manuel Cervera-Marzal y Eric Fabri (dirs.) (2015). Autonomie ou barbarie. La démocratie radicale de Cornelius Castoriadis et ses défis contemporains. Neuvy-en-Champagne: Le passager clandestin.

Turkle, S. (2017). En defensa de la conversación. Barcelona: Ático de libros.

Vázquez, A. (2014). Psicopolítica, sociedad sitiada y movimiento de los indignados: la modernidad líquida y sus parias. Zygmunt Bauman y Edward Snowden. Errancia 8. Disponible en http://www.iztacala.unam.mx/errancia/v10/litorales_11.html [2017, 12 de diciembre].

Winner, L. (1985). "Do Artifacts Have Politics?”, en: D. MacKenzie et al. (eds.), The Social Shaping of Technology, Philadelphia: Open University Press, 1985, [en línea]. Versión en castellano de Mario Francisco Villa. Disponible en http://www.oei.es/historico/salactsi/ winner.htm [2017, 17 de agosto].

Wittel, A. (2001). Toward a Network Sociality. In Theory, Culture \& Society. 18:51, [en línea]. Disponible en http://tcs.sagepub.com/content/18/6/51. 